\title{
SELFCONSISTENT CALCULATIONS OF HIGHLY EXCITED NUCLEI
}

\author{
M. BRACK and P. QUENTIN ${ }^{\ddagger}$ \\ The Niels Bohr Institute, University of Copenhagen, \\ $D K-2100$ Copenhagen $\phi$, Denmark
}

Received 20 June 1974

\begin{abstract}
Selfconsistent calculations of excitation energies and entropies of highly excited nuclei have been performed using the temperature-dependent constrained Hartree-Fock (HF)method with the Skyrme interaction. The selfconsistent variations of the level spectra as functions of the temperature (at a fixed deformation) are shown to be small and practically do not affect the results for the entropy versus excitation energy. The disappearance of the shell fluctuations in the potential energy surface of a rare earth nucleus around $T \sim 3 \mathrm{MeV}$ is demonstrated.
\end{abstract}

Several groups [1] have recently calculated level densities of highly excited nuclei. These calculations are done within the statistical model using the single particle spectrum of a deformed average nuclear potential. Starting from a deformation energy surface obtained with the Strutinsky method [2], the level density is evaluated as a function of deformation and of excitation energy. Ground-state or saddle point deformations have to be determined at each temperature, since the shell effects are known to disappear at high excitations $[2,3]$. However, the question remains to what extent the parameters of the average potential and of the liquid-drop model (LDM) should depend on the temperature. A possible way of answering this question is to use a selfconsistent approach which allows us to derive simultaneously the average potential, the single-particle states and the occupation probabilities at each temperature.

We present here some results of Hartree-Fock (HF) calculations at finite temperatures using the effective interaction of Skyrme which has been very successful in describing nuclear properties such as binding energies, radii and deformations [4]. This phenomenological interaction can be viewed [5] as an approximate effective interaction derived from $G$-matrix calculations [6] . Although the Goldstone--Bruckner approach has been generalized to the case of finite temperatures [e.g., 7], no numerical calculations of this kind have been undertaken so far. We have therefore

\footnotetext{
₹ Permanent address: Division de Physique Théorique, IPN Orsay, France.
}

to assume that the temperature dependence of the effective interaction can be neglected.

The derivation of the HF-approximation at finite temperature can be found in different textbooks [e.g., 8] and we quote here only those formulae which are relevant for our purpose. For simplicity we present the formalism without inclusion of pairing correlations. Since these are only important at low temperatures $(T \lesssim 1 \mathrm{MeV})$ which we are not especially interested in here, we have chosen temperatures for which the BCS gap is zero $(T \geqslant 1 \mathrm{MeV})$ in all our calculations. For $T=0$, however, we include the BCS pairing in a selfconsistent way as described in ref. [4] (see the case of ${ }^{168} \mathrm{Yb}$ below).

Starting from a many-body Hamiltonian $H=\mathcal{T}+\mathcal{V}$ with a nuclear interaction $\mathcal{\nu}=v^{(2)}+v^{(3)}+\ldots$, where $v^{(p)}$ acts between $p$ particles, one has to solve the coupled system of equations

$$
\begin{aligned}
& H(\rho) \varphi_{\nu}(r)=\epsilon_{\nu} \varphi_{\nu}(r), \\
& \operatorname{Tr} \rho=\sum_{\nu} f_{\nu}=\sum_{\nu}\left[1+\exp \left\{\beta\left(\epsilon_{\nu}-\mu\right\}\right]^{-1}=N,\right.
\end{aligned}
$$

where $N$ is the number of particles, $f_{\nu}$ are the Fermi occupation numbers, $\beta$ is the inverse temperature $(\beta=1 / k T)$ and $\mu$ the chemical potential. The onebody Hamiltonian $H(\rho)$ is defined as in usual HFtheory, but it depends now on the temperature through the density matrix $\rho$ which has the form

$$
\rho_{\alpha \beta}=\sum_{\nu} f_{\nu}\langle\alpha \mid \nu\rangle\langle\nu \mid \beta\rangle
$$


in terms of an arbitrary basis $|\alpha\rangle$. The HF equations $(1 \mathrm{a}, \mathrm{b})$ are obtained by minimizing the thermodynamical potential $\Omega=E-T S-\mu N$ with respect to $\varphi_{\nu}(r)$ and $f_{v}$. The total energy $E$ of the system is

$E=\langle\mathcal{T}+\mathcal{V}\rangle=\sum_{\nu} f_{\nu} \epsilon_{\nu}-\sum_{p}(p-1)\left\langle v^{(p)}\right\rangle$

and the entropy $S$ is

$S=-\sum_{\nu}\left\{f_{\nu} \ln f_{\nu}+\left(1-f_{\nu}\right) \ln \left(1-f_{\nu}\right)\right\}$.

The excitation energy $E^{*}(T)$ of the nucleus is defined at each temperature by $E^{*}(T)=E(T)-E(0)$.

We have performed calculations for the nuclei ${ }^{208} \mathrm{~Pb}$ and ${ }^{168} \mathrm{Yb}$ at temperatures up to $T=6 \mathrm{MeV}$.

For the parameters of the Skyrme interaction we use the set SIII which was used in recent extensive calculations [e.g., 9]. For nuclei at spherical symmetry we solve eq. (1a) in coordinate space. (We are grateful to $\mathrm{M}$. Beiner and $\mathrm{H}$. Flocard for providing us with an improved version of the spherical HF code by Vautherin and Brink [4]). For axially symmetric deformations, we diagonalize the HF-Hamiltonian in a truncated harmonic oscillator basis [4] .

At high temperatures $(T \gtrsim 2.5-3 \mathrm{MeV})$, one has to pay attention to the effects of unbound states in the continuum region, which we included in both codes. Moreover in de deformed code, the size of the basis and the optimization of its parameters become more and more crucial for $T>3 \mathrm{MeV}$. In order to check the importance of these effects, it is sufficient to compare the results obtained with the two different codes in a spherical case. We found that for ${ }^{208} \mathrm{~Pb}$ both the excitation energies $E^{*}$ and the entropies $S$ agree within $\sim 1 \%$ up to $T=2.5 \mathrm{MeV}\left(E^{*}=110 \mathrm{MeV}\right)$. (13 oscillator shells were included in the basis of the "deformed" code.) At larger temperatures, the results start slowly deviating, but in a way which does not affect the conclusions drawn in this paper. The numerical problems will be discussed in more detail in a forthcoming publication [10].

Physically, the process of nucleon evaporation becomes important at temperatures for which the occupation probabilities of unbound states are sufficiently large. Then the system starts dissociating and can no longer be described by equilibirum statistics. Our results at very high temperatures $(T \approx 5-6 \mathrm{MeV})$ do not take this effect into account and are therefore of academic interest.

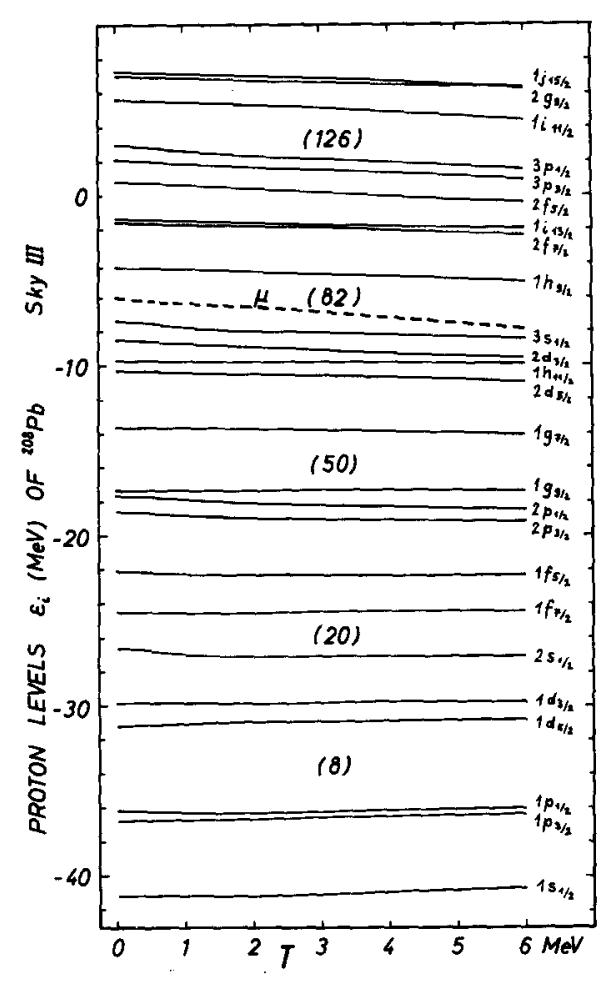

Fig. 1. Proton levels $\epsilon_{\nu}$ of ${ }^{208} \mathrm{~Pb}$ as functions of the temperature $T$. The dashed curve is the chemical potential $\mu$.

In fig. 1 we present the proton single-particle spectrum of ${ }^{208} \mathrm{~Pb}$ as a function of temperature. The levels $\epsilon_{\nu}$ appear to be remarkably constant. This can be understood qualitatively by looking at the behaviour of the potentials and the effective masses of the SkyrmeHF Hamiltonian (see ref. [4]). We find that the depths of the potentials remain essentially constant, whereas their r.m.s. radii in ${ }^{208} \mathrm{~Pb}$ increase by only $\sim 1 \%$ from $T=0$ to $T=2.5 \mathrm{MeV}$ and by $\sim 3-3.5 \%$ from $T=0$ to $T=6 \mathrm{MeV}$. On the other hand, the effective masses at the center increase respectively by $\sim 1.4-2 \%$ and $\sim 3 \%$. Both effects are due to the contributions from states of higher shells at larger temperatures. The effects are, however, not very large and tend to shift the position of the levels $\epsilon_{v}$ in opposite ways, thus partially cancelling each other.

It is interesting to see how these changes of the selfconsistent solutions affect the excitation energies and entropies. To do so, we compare the selfconsistent values $E^{*}$ (and $S$ ) with the approximate values $E^{*(0)}$ (and $S^{(0)}$ ) obtained by using the fixed ground- 


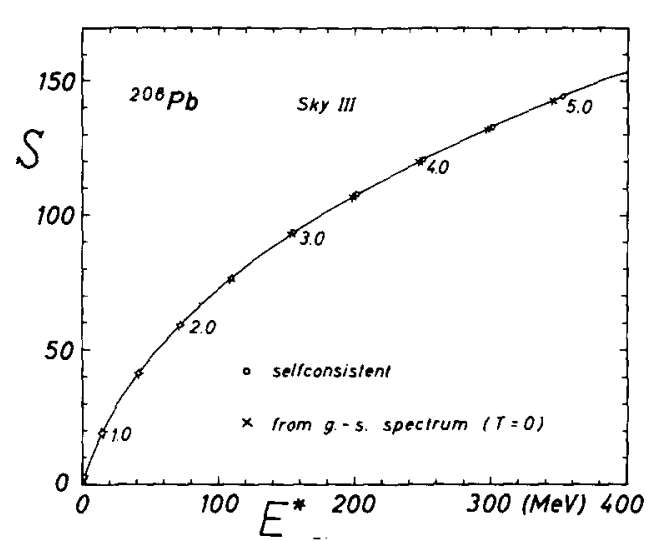

Fig. 2. Entropy $S$ versus excitation energy $E^{*}$ of ${ }^{208} \mathrm{~Pb}$, calculated at the temperatures indicated (in $\mathrm{MeV}$ ) along the curve. Circles show the selfconsistent values, crosses the approximations $E^{*}(0), S^{(0)}$ derived from the fixed ground-state spectrum (see eq. (5)).

state spectrum $\epsilon_{y}^{(0)}(T=0)$ and varying only the occupations $f_{v}$ with temperature. This approximation corresponds to the approach quoted above [1]. We define thus

$E^{*(0)}=\sum_{\nu} \epsilon_{\nu}^{(0)} f_{\nu}^{(0)}(T)-\sum_{\nu=1}^{N} \epsilon_{\nu}^{(0)}$,

$f_{\nu}^{(0)}(T)$ and $S^{(0)}$ are given by eqs. (4) and (1b) in terms of the $\epsilon_{\nu}^{(0)}$. The result of this comparison is shown in fig. 2 for ${ }^{208} \mathrm{~Pb}$. We see that the errors made in approximation (5) are negligible up to $T=2 \mathrm{MeV}$. What is even more remarkable is the fact that the approximated values $E^{*(0)}, S^{(0)}$ lie on the same curve $E^{*}(S)$ as the selfconsistent values, even up to $T=5$

$\mathrm{MeV}$. Thus the quantity of physical interest, namely the entropy as a function of excitation energy, is not affected at all by the change of the HF solutions with temperature. We conclude therefore that it is completely sufficient to use the fixed ground-state spectrum $\epsilon_{\nu}^{(0)}($ at $T=0)$ for calculating $E^{*}$ and $S$. Some preliminary calculations for other spherical nuclei lead us to the same conclusion (see also ref. [10]). Calculations of the level density $\rho\left(E^{*}\right)$, which depends in a more complicated way on the levels $\epsilon_{\nu}$, are in progress.

We shortly present some results for the rare-earth nucleus ${ }^{168} \mathrm{Yb}$ which has a prolate ground-state deformation with a mass quadrupole moment $Q_{2}$ of

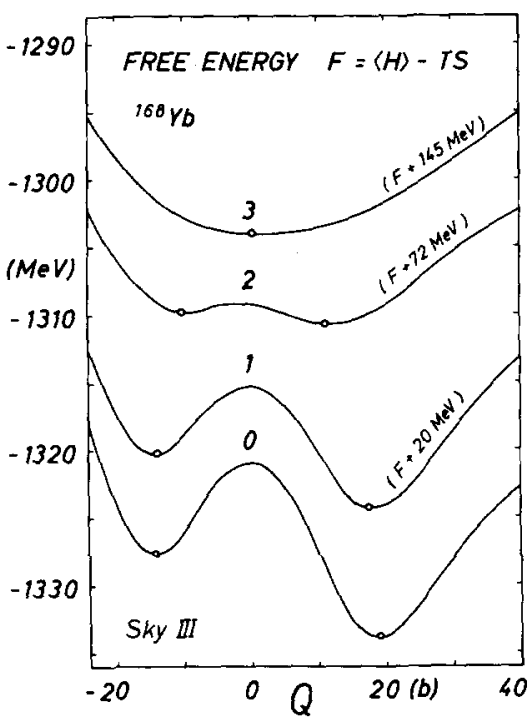

Fig. 3. Potential (free) energy of ${ }^{168} \mathrm{Yb}$ versus mass quadrupole moment at different temperatures $(T=0,1,2,3 \mathrm{MeV})$. The curves with $T>0$ are shifted upwards by the amount indicated in parentheses. Pairing included.

18.1 barns. We included 11 oscillator shells in the deformed basis. The convergence of the results was checked by calculating some cases with 13 shells. Fig. 3 shows the deformation energy curves of ${ }^{168} \mathrm{Yb}$ at different temperatures. The quantity plotted is the free energy $F=E-T S$ versus the mass quadrupole moment $Q_{2}$. The local minima of the curves, indicated by circles, were obtained without constraint. For the other points a quadratic constraint on the quadrupole moment was used (see Flocard et al. [4]). At $T=0$ the pairing effects were included selfconsistently in the BCS-approximation [4] using constant pairing strengths. We checked that the critical temperature $T_{0}$ at which the gaps disappear is less than $1 \mathrm{MeV}$ for all deformations considered.

For a fixed value of the quadrupole moment, the approximation (5) for $E^{*(0)}$ and $S^{(0)}$ was found to be just as good for ${ }^{168} \mathrm{Yb}$ as for ${ }^{208} \mathrm{~Pb}$. We can conclude that the temperature dependence of the HF spectra $\epsilon_{\nu}$ can be neglected for a fixed deformation of the nucleus. This result justifies $a$ posteriori the non-selfconsistent approach [1] in which the spectra $\epsilon_{\nu}$ are held constant.

Thus the disappearance of the shell structure with 
increasing temperature, which manifests itself in fig. 3, is in agreement with earlier results [1]. The equilibrium deformations are almost not affected up to $\sim 1$ $\mathrm{MeV}$. At larger temperature they decrease and at $T \approx$ $3 \mathrm{MeV}$, where the energy surface is close to a LDM surface, the minimum is at a spherical shape. Of course our approach is a purely static one. Due to the increasing softness of the spherical barrier, an inclusion of dynamics which would allow collective vibrations of the nucleus could easily lead to an average spherical shape already at $T \approx 2 \mathrm{MeV}$. The shape of the curves $F(Q)$ at $T \gtrsim 3 \mathrm{MeV}$ can be used to extract information about the temperature dependence of the LDM parameters inherent in the interaction used. Such an investigation is in progress.

More detailed results for the two nuclei considered here, as well as for the superheavy nucleus with $Z=$ $114, A=298$, are presented in ref. [10].

We are grateful to Professor B. Mottelson and Drs. S. Bjфrnholm, A.S. Jensen and P. Siemens for enlightening discussions. We are especially indebted to Professors M. Vénéroni and A.D. Jackson for valuable criticism of the manuscript. The hospitality extended to us at the Niels Bohr Institute together with the support from the Japan World Exposition Commemorative Fund is gratefully acknowledged.

While this paper was being prepared, our attention was drawn to a preprint by Mosel, Zint and Passler (Giessen University), who did similar calculations for lighter nuclei. Their results for ${ }^{40} \mathrm{Ca}$, showing a strong variation of the levels $\epsilon_{\nu}$ with temperature and a clear discrepancy between the curves $S\left(E^{*}\right)$ and $S^{(0)}\left(E^{*}(0)\right.$ above $T \approx 2 \mathrm{MeV}$, are in contradiction with our find- ings. We have therefore done a calculation for ${ }^{40} \mathrm{Ca}$ with the spherical code. The result entirely confirms the conclusions drawn above from the ${ }^{208} \mathrm{~Pb}$ case.

\section{References}

[1] V.S. Ramamurthy et al., Phys. Rev. Lett. 25 (1970) 386 ;

L. Moretto, Nucl. Phys. A182 (1972) 641;

J.R. Huizenga and L. Moretto, Am. Rev. Nucl. Sci. 22 (1972) 427;

A.S. Jensen and J. Damgaard, Nucl. Phys. A210 (1973) 282;

T. D $\phi$ ssing and A.S. Jensen, Nucl. Phys. A222 (1974) 493.

[2] V.M. Strutinsky, Nucl. Phys. A95 (1967) 420; A122 (1968) 1;

M. Brack et al., Rev. Mod. Phys. 44 (1972) 320.

[3] A. Bohr and B. Mottelson, Nuclear Structure, Vol. 2, to be published.

[4] D. Vautherin and D.M. Brink, Phys. Rev. C5 (1972) 626;

D. Vautherin, Phys. Rev. C7 (1973) 296;

H. Flocard et al., Nucl. Phys. A203 (1973) 433.

[5] J. Negele and D. Vautherin, Phys. Rev. C5 (1972) 1472.

[6] J. Negele, Phys. Rev. C1 (1970) 1260.

[7] C. Bloch, Studies in statistical mechanics, Vol. III, pp. 3-211, eds. J. De Boer and G.E. Uhlenbeck (NorthHolland, Amsterdam, 1965).

[8] D.J. Thouless, The quantum mechanics of many-body systems (Academic Press, 1961);

J. des Cloiseaux, Many-body physics, Les Houches 1967, pp. 1-36, eds. C. de Witt and R. Balian (Gordon and Breach, 1968).

[9] M. Beiner et al., to be submitted to Nuclear Physics.

[10] M. Brack and O. Quentin, Nobel Symposium on Superheavy Elements, Ronneby, Sweden, 1974, to be published in Physica Scripta. 